Spectrum Disorder was typified by VS hypoactivation to social and non-social reward, while Psychopathy was associated with VS hyperactivation in response to non-social reward anticipation. No studies were identified of social reward in Psychopathy.

Conclusion. The reported fMRI findings correlate with clinical observations in both conditions. Reduced reward response in ASD to a range of social and non-social stimuli would provide a parsimonious account of the social and non-social deficits that characterise the condition. Enhanced responses to the anticipation of reward in Psychopathy provides an account of the ruthless and destructive pursuit of reward-driven behaviours not inhibited by immoral or aversive signals. If, as the literature suggests, reward circuitry dysfunction plays a role in the development and manifestation of symptoms in both conditions, reward processing and its underlying neural circuitry may represent important targets for the development of novel treatment strategies.

\section{The utility of the Brief Edinburgh Depression Scale (BEDS) in assessing severity of depression in advanced cancer patients}

\author{
Zeryab Meyer ${ }^{1 \star}$, Christopher Shiels ${ }^{2}$, Christopher Dowrick ${ }^{3}$ \\ and Mari Lloyd-Williams ${ }^{4}$ \\ ${ }^{1}$ School of Medicine, University of Liverpool; ${ }^{2}$ Research Associate, \\ Institute of Population Health Sciences, University of Liverpool; \\ ${ }^{3}$ Professor of Primary Medical Care, Institute of Population Health \\ Sciences, University of Liverpool and ${ }^{4}$ Professor and Honorary \\ Consultant in Palliative Medicine, Academic Palliative and Supportive \\ Care Studies Group (APSCSG), University of Liverpool \\ ${ }^{*}$ Corresponding author.
}

doi: 10.1192/bjo.2021.161

Aims. When using an assessment tool, brevity and validity are essential. Although brief depression inventories exist, they rely heavily on the inclusion of somatic symptoms. This can be problematic in advanced cancer populations; weight loss and sleep disturbance are for the most part ubiquitous in these patients and may not necessarily be indicative of depression.

The Brief Edinburgh Depression Scale (BEDS) is a 6-item shortened version of the Edinburgh Depression Scale which has been validated for use in patients with advanced cancer and is used internationally. The BEDS cut off threshold of 6/18 indicates that depression may be present. However, the BEDS currently provides no information regarding severity. The aim of this study is to establish severity thresholds for the BEDS by comparing it to another depression scale: the commonly used, rigorously validated, Patient Health Questionnaire (PHQ-9).

Method. 284 advanced cancer patients attending hospice day services in the North West of England completed both the PHQ-9 and the BEDS. Mean participant age was 66.7 (Standard Deviation $=13.2)$ and the sample contained both males $(\mathrm{n}=$ $102,36 \%)$ and females $(n=182,64 \%)$. BEDS severity thresholds with the highest Sensitivity (Sn) and Specificity (Sp) were selected based on their ability to predict PHQ-9 categories.

Result. A BEDS score of 4 to 6 was selected to indicate 'mild depression' $(\mathrm{Sn}=81.7, \mathrm{Sp}=65)$; 7 to 8 'moderate depression' $(\mathrm{Sn}=74.8, \mathrm{Sp}=78.7) ; 9$ to 11 'moderately severe depression' $(\mathrm{Sn}=82, \mathrm{Sp}=82.9)$ and 12 or more 'severe depression' $(\mathrm{Sn}=$ 63.2, $\mathrm{Sp}=92.8$ ). A linearly weighted kappa (with $\mathrm{s}$ weighting) showed a moderate level of agreement $(0.47,95 \%$ Confidence Interval: 0.40-0.54).

Conclusion. The BEDS is a simple and brief tool used to screen for depression in advanced cancer patients. It is administered throughout the UK and multiple translation studies have enabled its global a (including in resource poor countries). The severity thresholds calculated here are derived from a large sample of patients with advanced cancer attending hospice services and demonstrate acceptable sensitivity and specificity in relation to the PHQ-9, a thoroughly validated reference standard. We conclude that the generated BEDS thresholds support use of the BEDS in determining the presence and severity of depression in advanced cancer populations.

\section{Improving facilitation of ECT treatment for patients in an acute medical hospital}

\section{Vatsala Mishra*, Chun Chiang Sin Fai Lam, Marilia Calcia} and Isabel McMullen

Mental Health Liaison Team, King's College Hospital, South London and Maudsley NHS Foundation Trust

${ }^{*}$ Corresponding author.

doi: 10.1192/bjo.2021.162

Aims. A Quality Improvement Project aiming to streamline facilitation of electroconvulsive therapy (ECT) treatment for psychiatric patients at a general acute hospital and reduce cancellation rates via the use of a checklist.

ECT treatment is an essential aspect of psychiatric care for patients with severe depression or treatment-resistant psychosis. Facilitation of ECT treatment is an uncommon task for liaison psychiatry and the medical and nursing teams responsible for patients' medical care. Between August-October 2019, this liaison psychiatry team had 3 patients undergoing ECT treatment a total of 13 times, with treatment being cancelled on 4 occasions. After engagement with stakeholders from the acute medical teams, the liaison team and the ECT suite team, key areas requiring intervention were identified to help reduce the rates of cancellation. Areas identified included a lack of ownership on the logistic and operational aspects of ECT amongst staff, a lack of knowledge of what the process involved and a lack of confidence in managing said patients. Difficulties in communication between teams and accurate documentation may contribute to errors and cancellation of ECT sessions, which in turn would delay treatment and impact on patient safety and clinical outcomes.

Method. The first author, a Foundation Year 1 doctor, developed a 10-point checklist to be referred to when arranging ECT for patients, to ensure errors were not made which could lead to missed treatment and delayed recovery. The tasks and responsibilities of each key member of the team were clearly identified. This checklist was included in all ECT patients' files and teaching was provided to staff involved. Feedback was obtained from staff involved regarding the clarity of information and their confidence in managing such cases.

Result. In the month following initial intervention the liaison psychiatry team organised 12 ECT sessions. The checklist was pasted into notes the day before each ECT session and 0 sessions were missed for avoidable reasons. Feedback from staff showed all teams felt more confident co-ordinating ECT treatment as a result of the checklist.

Conclusion. Creating a 10-point checklist for the facilitation of ECT treatment in patients at a medical hospital was beneficial in reducing avoidable errors from $16 \%$ to $0 \%$. The liaison psychiatrists, medical doctors, and nurses involved reported greater confidence in managing patients undergoing ECT and described the checklist as enhancing the feeling of teamwork and communication within the multi-disciplinary team, and felt it had improved patient safety and clinical outcomes. 
War-related trauma and post-traumatic stress disorder prevalence among Syrian university students

Fatema Mohsen ${ }^{1 \star}$, Yousef Latifa ${ }^{2}$ and Bisher Sawaf ${ }^{3}$

${ }^{1}$ Syrian Private University; ${ }^{2}$ Damascus University and ${ }^{3} \mathrm{Hamad}$

medical corporation

${ }^{*}$ Corresponding author.

doi: 10.1192/bjo.2021.163

Aims. PTSD is one of the most prevalent mental disorders in waraffected regions. Syria has endured 10 years of war and yet little is known about the impact of the conflict on the well-being of Syrians who remain. This study aimed to provide an estimated prevalence of PTSD among trauma-exposed university students in Deir-ez-Zor, Syria, a war-ridden region, that was under siege by the Islamic State of Iraq and the Levant (ISIS) for over 3 years. Moreover, we aimed to study the different types of trauma to which the students were exposed and studied the association between PTSD and multiple covariates including, socio-demographic characteristics, smoking habits, academic performance, and stress levels, and identify factors that influence the development of PTSD symptoms.

Method. A descriptive cross-sectional study design was used on a sample of Al-Furat university students in Deir-ez-Zor. We collected data on socio-demographics, trauma exposure, and stress levels. PTSD Checklist for DSM-5 was used to carry out PTSD diagnosis and to determine the severity of the disorder.

Result. A total of 833 Syrian students were recruited into the study, the mean was $22.4 \pm 3.2$ years. Of those, $(22.2 \%)$ have been displaced 3 times, while (18.8\%) were displaced over 5 times. (86.4\%) reported experiencing at least one traumatic event, $(33.8 \%)$ of the participants were exposed to one traumatic event, and (44.7\%) experienced four or more traumatic events. PTSD prevalence was (28.2\%), and the highest PTSD rates were found among students who were forced into sexual acts (46.3\%), followed by those who witnessed childhood trauma or violence and those who witnessed violence as adults (42.6\%). Sample distribution over stress levels was as follows: normal (39.5\%), mild (16.0\%), moderate (17.8\%), severe (17.3\%), and extremely severe (9.8\%). A statistically significant association was found between PTSD prevalence and stress severity $(\mathrm{p}=0.000)$. A significant association was found between PTSD and internal displacement $(p=0.032)$, academic year $(p=0.002)$, and social-economic status $(p=0.000)$. Binary logistic regression revealed that smokers (vs non-smokers, $\mathrm{OR}=0.259, \mathrm{p}$ $=.034$ ) and third-year students (vs fifth year, $\mathrm{OR}=0.44, \mathrm{p}=.019$ ) were significantly associated with PTSD.

Conclusion. The results presented in this research revealed a high prevalence of trauma exposure and PTSD among a sample of university students in Deir-ez-Zor. These findings call for immediate actions to help the affected population in restoring their mental health, so they can be prepared to face the challenges and demands of the post-conflict period.

Sleep quality and adherence to medical therapy among hemodialysis patients with depression: a cross-sectional study from a developing country

Syed Muhammad Jawad Zaidi, Mehwish Kaneez, Javeria Awan, Hamza Waqar Bhatti, Muhammad Hamza, Mishal Fatima and Muhammad Zubair Satti

Rawalpindi Medical University

${ }^{\star}$ Corresponding author.

doi: 10.1192/bjo.2021.164

Aims. Depression is a fairly common finding among end-stage renal disease (ESRD) patients on hemodialysis and is an independent risk factor for morbidity and mortality. The psychiatric manifestations of the disease may affect their compliance to medications and alter sleep quality that is often overlooked by nephrologists. This translates into poor quality of life and poorer disease prognosis. Thus, Our study aims to assess the prevalence of depression and its association with compliance to medical therapy and sleep quality among ESRD patients on hemodialysis.

Method. In this cross-sectional study, a total of 288 hemodialysis patients with a confirmed diagnosis of ESRD were evaluated for depression using Patient Health Questionnaire-9 (PHQ-9) scale. Only the patients with moderate to severe depressive symptoms on PHQ-9 were further evaluated for sleep quality and compliance to medications using the Pittsburgh Sleep Quality Index (PSQI) and Drug Attitude Inventory-10 (DAI-10) respectively. The characteristics of ESRD patients with depression were also assessed. Median PHQ-9, DAI-10, and PSQI scores were calculated and the correlation between study variables was assessed using spearman's correlation.

Result. Of the 288 included participants, 188 (65.27\%) had depression as evaluated via PHQ-9. Of these 188 patients, 114 were males while 74 were females. A total of $113(60.01 \%)$ of the depressed patients had poor compliance with medication while $137(72.87 \%)$ patients had poor sleep quality. Higher PHQ-9 scores were positively correlated with disease duration, dialysis years, and time between diagnosis and therapy $(r=0.41$, 0.39 , and 0.43 respectively) and negatively with marital and employment status $(\mathrm{r}=-0.32$ and -0.49 respectively). Spearman's correlation matrix showed that PHQ-9 scores were negatively correlated with DAI-10 but positively correlated with PSQI scores.

Conclusion. The study indicates a high prevalence of depression among ESRD patients on hemodialysis. Poor sleep quality and non-adherence to medications are extremely common among ESRD patients with depression. These psychiatric components must be considered to optimize medical treatment and improve the quality of life in this subset of patients.

\section{Exploring the predictive factors for depression among hemodialysis patients: a case-control study \\ Syed Muhammad Jawad Zaidi ${ }^{\star}$, Mehwish Kaneez ${ }^{2}$, Hamza Waqar Bhatti ${ }^{2}$, Shanzeh Khan ${ }^{2}$, Shafaq Fatima ${ }^{2}$, Muhammad Hamza ${ }^{2}$ and Mishal Fatima ${ }^{2}$ \\ ${ }^{1}$ Final Year MBBS Student, Rawalpindi Medical University and \\ ${ }^{2}$ Rawalpindi Medical University \\ ${ }^{\star}$ Corresponding author.}

doi: 10.1192/bjo.2021.165

Aims. Depression remains an exceedingly ubiquitous entity that significantly depreciates the quality of life and disease prognosis among end-stage renal disease (ESRD) patients. Even though the deleterious effects of depression on ESRD patients are well-established in the literature, the predictive factors that predispose such patients to depression need to be explored. Our study thus aims to gauge these factors and create a predictive model for optimal psychiatric and medical management of such patients. Method. All ESRD patients with a disease duration of at least one year underwent a complete psychiatric evaluation based on DSM-V guidelines preceded by a cognitive evaluation by Mini-Mental State Examination (MMSE). A total of 73 patients diagnosed with moderate to severe major depressive disorder were selected as cases. Patients suffering from recurrent psychotic episodes, having a past or family history of psychiatric illness, being already treated for depression, having any substance abuse (current or past), were excluded from the study. Following the similar guidelines, and exclusion criteria, 146 patients (two controls for 